\title{
Sleep disordered breathing and enlargement of the right heart after myocardial infarction
}

\author{
Stefan Buchner ${ }^{1,5}$, Michael Eglseer ${ }^{1,5}$, Kurt Debl ${ }^{1}$, Andrea Hetzenecker ${ }^{1}$, \\ Andreas Luchner ${ }^{1}$, Oliver Husser ${ }^{1}$, Christian Stroszczynski², Okka W. Hamer ${ }^{2}$, \\ Claudia Fellner ${ }^{2}$, Florian Zeman ${ }^{3}$, Michael Pfeifer ${ }^{1,4}$ and Michael Arzt ${ }^{1}$
}

Affiliations: ${ }^{1}$ Klinik und Poliklinik für Innere Medizin II, Universitätsklinikum Regensburg, Regensburg, Germany. ${ }^{2}$ Institut für Röntgendiagnostik, Universitätsklinikum Regensburg, Regensburg, Germany. ${ }^{3}$ Zentrum für klinische Studien, Biostatistics, Universitätsklinikum Regensburg, Regensburg, Germany. ${ }^{4}$ Zentrum für Pneumologie, Klinik Donaustauf, Donaustauf, Germany. ${ }^{5}$ Both authors contributed equally.

Correspondence: Stefan Buchner, Klinik und Poliklinik für Innere Medizin II, Universitätsklinikum Regensburg, Franz-Josef-Strauss-Allee 11, 93053 Regensburg, Germany.

E-mail: stefan.buchnerdukr.de

ABSTRACT Structural and functional integrity of the right heart is important in the prognosis after acute myocardial infarction (AMI). The objective of this study was to assess the impact of sleep disordered breathing (SDB) on structure and function of the right heart early after AMI.

54 patients underwent cardiovascular magnetic resonance 3-5 days and 12 weeks after AMI, and were stratified according to the presence of SDB, defined as an apnoea-hypopnoea index of $\geq 15$ events $\cdot \mathrm{h}^{-1}$.

12 weeks after AMI, end-diastolic volume of the right ventricle had increased significantly in patients with $\operatorname{SDB}(n=27)$ versus those without $(\mathrm{n}=25)$ (mean \pm SD $14 \pm 23 \%$ versus $0 \pm 17 \%, \mathrm{p}=0.020)$. Multivariable linear regression analysis accounting for age, sex, body mass index, smoking, left ventricular mass and left ventricular end-systolic volume showed that the apnoea-hypopnoea index was significantly associated with right ventricular end-diastolic volume (B-coefficient 0.315 (95\% CI 0.013-0.617); $\mathrm{p}=0.041$ ). From baseline to 12 weeks, right atrial diastolic area increased more in patients with $\operatorname{SDB}\left(2.9 \pm 3.7 \mathrm{~cm}^{2}\right.$ versus $1.0 \pm 2.4 \mathrm{~cm}^{2}$, $\mathrm{p}=0.038$; when adjusted for left ventricular end systolic volume, $\mathrm{p}=0.166$ ).

SDB diagnosed shortly after AMI predicts an increase of right ventricular end-diastolic volume and possibly right atrial area within the following 12 weeks. Thus, SDB may contribute to enlargement of the right heart after AMI.

@ERSpublications

Sleep disordered breathing may predispose to enlargement of the right heart after acute myocardial infarction http://ow.ly/Cc6vv

Received: March 252014 | Accepted after revision: Sept 052014 | First published online: Oct 302014

Support statement: The study was funded by ResMed (Martinsried, Germany), Philips Home Healthcare Solutions (Murrysville, PA, USA) and the Faculty of Medicine of the University of Regensburg (Regensburg, Germany).

Conflict of interest: Disclosures can be found alongside the online version of this article at erj.ersjournals.com

This article has supplementary material available from erj.ersjournals.com

Copyright OERS 2015 


\section{Introduction}

Right ventricular (RV) dysfunction occurs in approximately $20 \%$ of patients with acute myocardial infarction (AMI) and impaired left ventricular (LV) systolic function [1]. Structural and functional integrity of the right heart is important in the prognosis after AMI [1, 2]. Long-term survival is significantly lower in patients with persistent RV dysfunction 3 months after AMI, and RV dysfunction is associated with a higher end-diastolic dimension of the right ventricle [2]. Furthermore, RV systolic dysfunction is an independent predictor of the development of heart failure and mortality after AMI [1].

Sleep disordered breathing (SDB) is present in $43-66 \%$ of patients with AMI [3-6]. AMI patients with SDB are exposed to repetitive hypoxia, increased heart rate and increased cardiac afterload [7]. In addition, coronary blood flow is uncoupled from cardiac workload in patients with SDB and related endothelial dysfunction [8]. Through these mechanisms, SDB contributes to reduced myocardial salvage, increased infarct size and impaired recovery of LV function in the early phase after AMI $[3,9]$. Furthermore, SDB can specifically affect the right heart via hypoxia-induced increases in pulmonary artery pressure [10-12]. Reduced nitric oxide-mediated vasodilation of the pulmonary vessels may contribute to increased pulmonary artery pressure in patients with SDB [13].

Cardiovascular magnetic resonance (CMR) has been used to assess the influence of SDB on the right ventricle of patients who do not have overt cardiac disease. Two recent uncontrolled trials using CMR concluded that continuous positive airway pressure treatment in otherwise healthy patients with moderate-to-severe SDB significantly reduced RV volume [14, 15], implying that SDB exerts adverse effects on the right ventricle, even in the absence of other cardiovascular comorbidities.

However, the influence of SDB on RV function has not yet been studied in AMI patients. Therefore, the aim of this study was to evaluate whether the presence of SDB in patients with AMI influences the structure and function of the right heart, as assessed by CMR.

\section{Methods}

Patients

Inclusion criteria were as follows: patients aged 18-80 years with a first-time AMI (ST elevation on ECG or complete occlusion of the coronary artery in non-ST elevation myocardial infarction) and percutaneous coronary intervention (PCI) who were treated at the University Hospital Regensburg (Regensburg, Germany) within $24 \mathrm{~h}$ of symptom onset. Exclusion criteria were as follows: previous myocardial infarction or previous myocardial revascularisation (PCI or surgical); indication for surgical myocardial revascularisation; cardiogenic shock; implanted cardiac device or other contraindications for CMR; known treated SDB; lung disease; other severe diseases (e.g. stroke); and unfeasible follow-up (e.g. length of distance to place of residence, language, etc.).

\section{Study design}

This prospective observational study was performed at the Universitätsklinikum Regensburg (Regensburg, Germany). The study protocol was reviewed and approved by the local institutional ethics committee. The study was performed according to the Helsinki Declaration of Good Clinical Practice. Written informed consent was obtained from all patients prior to enrolment. Eligible patients underwent an overnight in-laboratory sleep study (polysomnography) 3-5 days after PCI. CMR studies were performed 3-5 days and 12 weeks after PCI. Clinical management and medication was at the discretion of the responsible physician according to current practice and guidelines.

Patients were stratified into those with SDB (SDB group) (apnoea-hypopnoea index (AHI) of $\geq 15$ events $\mathrm{h}^{-1}$ ) and those without SDB (no SDB group) (AHI $<15$ events $\mathrm{h}^{-1}$ ). An AHI of $\geq 15$ events $\cdot \mathrm{h}^{-1}$ indicated at least a moderate degree of SDB [16]. None of the included patients was treated with positive airway pressure therapy within the first 12 weeks of AMI. In a subanalysis, the SDB group was stratified into patients with predominantly central apnoeas and hypopnoeas (central sleep apnoea (CSA)) and those with obstructive sleep apnoea (OSA). Another subgroup analysis was performed that only included patients with $\mathrm{RV}$ involvement in myocardial infarction determined by analysis of late-enhancement CMR images.

\section{Polysomnography}

Polysomnography was performed in all subjects using standard polysomnographic techniques (Alice System; Respironics, Pittsburgh, PA, USA) [6]. The sleep lab is located on the cardiology ward of the hospital to which participants were admitted with AMI. The median times to baseline and follow-up polysomnography after AMI were 3 days and 12 weeks, respectively. Respiratory efforts were measured with the use of respiratory inductance plethysmography, and airflow was measured using a nasal pressure cannula. Sleep stages, arousals, and apnoeas and hypopnoeas were determined according to the criteria of the American Academy of Sleep Medicine [17] by one experienced sleep technician blinded to the clinical data. 
Apnoea was defined as a cessation of inspiratory airflow for $\geq 10$ s. Hypopnea definition A was used ( $\geq 30 \%$ airflow reduction and $\geq 4 \%$ desaturation) [17]. AHI was defined as the number of apnoeas and hypopnoeas per hour of sleep.

\section{CMR acquisition protocol}

CMR studies were performed using a clinical 1.5-Tesla scanner (Avanto; Siemens Healthcare Sector, Erlangen, Germany) with a 32-channel phased-array receiver coil. Examination of ventricular function was performed using acquisition of steady-state free precession cine images in standard short axis planes (slice thickness $8 \mathrm{~mm}$, inter-slice gap $2 \mathrm{~mm}$, repetition time $60.06 \mathrm{~s}$, echo time $1.16 \mathrm{~s}$, flip angle $60^{\circ}$, field of view $300 \times 300 \mathrm{~mm}$, matrix size $134 \times 192$ pixels, readout pixel bandwidth $930 \mathrm{~Hz}$ per pixel). Delayed enhancement images were obtained using a segmented inversion recovery steady-state free precession technique (slice thickness $8.0 \mathrm{~mm}$, inter-slice gap $2 \mathrm{~mm}$, repetition time $1 \mathrm{RR}$ interval, echo time $1.48 \mathrm{~ms}$, flip angle $60^{\circ}$, field of view $360 \times 360 \mathrm{~mm}$, matrix size $128 \times 256$ ) and acquired $10-15 \mathrm{~min}$ after injection of gadolinium.

\section{CMR image analysis}

Evaluation of RV volume and RV ejection fraction (RVEF) was performed in the serial short axis slices using commercially available software (Syngo Argus, version B15; Siemens Healthcare Sector). In order to determine the volume of the right ventricle, endocardial contours of the right ventricle were traced manually for each short axis slice, from the most basal slice at the level of the cardiac valves to the most apical slice. These tracings were performed both at the end-diastolic and the end-systolic phases of the ventricular contraction.

RV tricuspid annular plane systolic excursion (TAPSE) was determined in the four-chamber view by tracing the point of insertion of the tricuspid valve onto the free wall of the right ventricle at end-diastole and end-systole of the right ventricle. Both insertion points were then transferred to a single four-chamber view in order to calculate the distance of these two points in respect to each other in centimetres. TAPSE can be interpreted as a semi-quantitative evaluation of RVEF overall [18]. RV infarct size was assessed manually using planimetry on short-axis slices, delineating hyper-enhanced areas.

The four-chamber view was also used to assess the area of the right and left atria. At the end of the diastolic and systolic phase of the atrial contraction, the free-walls, the atrial septum and the tricuspid or mitral valve were traced manually in order to determine the area of the atria. The adequate four-chamber view was chosen based on the following criteria: 1) all boundaries of the atria were represented; and 2) the atrial and ventricular septum were clearly depicted in their full extent on the selected slice. The diastolic and systolic area of the right and left atria where then used to determine the right atrial fractional area change and the left atrial fractional area change. Fractional area change represents the change in area during the contraction of the atria as a percentage.

\section{Statistical analysis}

Data are expressed as mean $\pm \mathrm{SD}$, unless otherwise indicated. Differences between groups were assessed using two-sided t-tests for continuous variables and Chi-squared tests for nominal variables. If the expected counts were $<5$, the Fisher's exact test was used.

Simple linear regression models were performed to assess the impact of baseline AHI on RV volume and the right atrial area. To control for potential confounders, such as age, sex, body mass index (BMI), smoking, LV mass and LV end-systolic volume (LVESV), additional multivariable linear regression models were calculated [19].

ANCOVA was used to compare changes in variables over time between the two patient groups. p-values for between-group differences were adjusted for LVESV using linear regression analysis. All reported p-values are two-sided, and a p-value of 0.05 was considered the threshold for statistical significance. Data entry and calculations were made using SPSS 19.0 (IBM Corp., Armonk, NY, USA).

\section{Results}

\section{Patient characteristics}

A total of 252 consecutive patients with first-time AMI who received PCI were evaluated for eligibility to be included in this study. 74 patients fulfilled the inclusion criteria and the exclusion criteria and were able to give written informed consent. 20 patients had to be excluded from the analysis due to withdrawal of consent for CMR or polysomnography, or because of an inability to analyse RV function. 54 patients were included in the final analysis and were stratified on the basis of the presence or absence of SDB on polysomnography (SDB group $n=29$; no SDB group $n=25$ ) (fig. 1). 


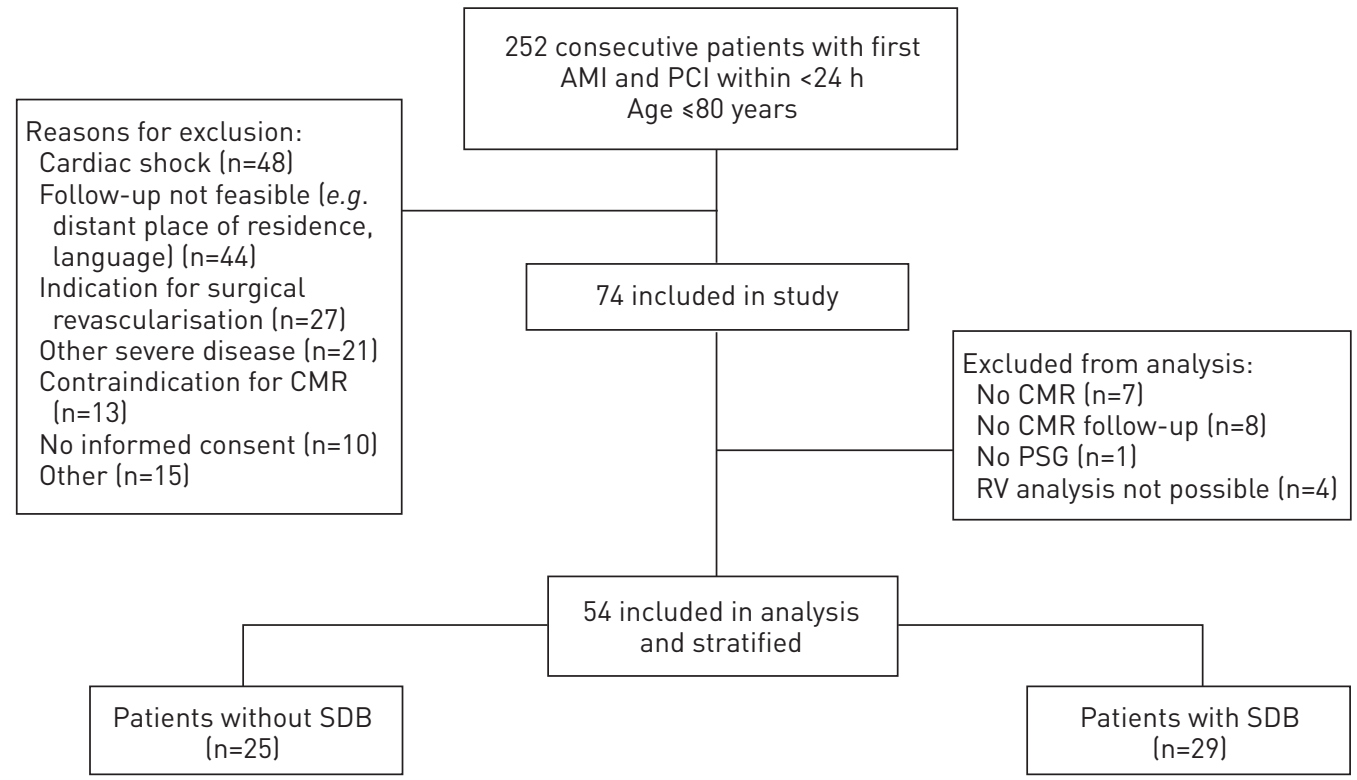

FIGURE 1 Flow of patients through the study. AMI: acute myocardial infarction; PCI: percutaneous coronary intervention; CMR: cardiac magnetic resonance; SDB: sleep disordered breathing; PSG: polysomnography; RV: right ventricular.

BMI was significantly higher in the SDB group compared with the no SDB group (table 1). There were no significant differences between the two patient groups with respect to age, sex and pre-existing cardiovascular risk factors, such as hypertension, diabetes and hypercholesterolaemia; the proportion of smokers was higher in the no SDB group (table 1).

The majority of AMIs were ST-elevation myocardial infarctions, and the proportion of left anterior descending (LAD) and non-LAD infarctions was similar in the two groups. Thrombolysis in myocardial infarction flow before and after PCI, Killip classification, heart rate, and systolic and diastolic blood pressure were similar in the SDB and no SDB groups.

LVESV was significantly greater and LV ejection fraction tended to be lower in the SDB compared with the no SDB group (table S1 of the online supplementary material). All patients received similar medication for the management of AMI (table 1).

The prevalence of SDB (an AHI of $\geq 15$ events.h ${ }^{-1}$ ) was $54 \%$ at baseline and decreased to $40 \%$ after 12 weeks $(\mathrm{p}=0.63$ ). Patients with SDB had severe disease (table 2 ), with $48 \%$ having predominantly central apnoeas and hypopnoeas. In the SDB group, AHI and central AHI decreased significantly from medians of 30 to 18 events $h^{-1}(p=0.003)$ and from 8 to 6 events $h^{-1}(p=0.005)$, respectively. Minimum oxygen saturation was lower in the SDB group versus the no SDB group. Both the SDB and no SDB groups showed similarly impaired sleep efficiency $<5$ days after AMI, while the proportion of rapid eye movement sleep was reduced to a significantly greater extent in the SDB group (table 2).

There were no significant changes in BMI in both groups between baseline and 12-week follow-up.

\section{Left atrial parameters}

Left atrial systolic area (LA-SA) was significantly larger in patients from the SDB group compared with the no-SDB group both at baseline $\left(18.2 \pm 5.3 \mathrm{~cm}^{2}\right.$ versus $\left.15.0 \pm 3.7 \mathrm{~cm}^{2}, \mathrm{p}=0.017\right)$ and after 12 weeks $\left(17.2 \pm 4.8 \mathrm{~cm}^{2}\right.$ versus $14.2 \pm 4.2 \mathrm{~cm}^{2}, \mathrm{p}=0.022$ ). After accounting for LVESV, the LA-SA at baseline and after 12 weeks was not significantly different between groups $(\mathrm{p}=0.194$ and $\mathrm{p}=0.155$, respectively). The left atrial diastolic area at baseline $\left(25.8 \pm 5.9 \mathrm{~cm}^{2}\right.$ versus $\left.23.2 \pm 5.4 \mathrm{~cm}^{2}, \mathrm{p}=0.107\right)$ and after 12 weeks $\left(25.7 \pm 4.9 \mathrm{~cm}^{2}\right.$ versus $23.1 \pm 4.8 \mathrm{~cm}^{2}$, $\mathrm{p}=0.059$ ) did not differ significantly between patient groups. Furthermore, the left atrial fractional area change was lower in the SDB group compared with the no SDB group at baseline and at 12 weeks $(29.8 \pm 9.5 \%$ versus $35.1 \pm 7.2 \%, \mathrm{p}=0.030$, and $33.3 \pm 10.9 \%$ versus $39.2 \pm 9.1 \%, \mathrm{p}=0.043$, respectively).

\section{$R V$ parameters}

At baseline, RV end-diastolic volume (RVEDV) and RV end-systolic volume (RVESV) were similar in both groups (table 3). RVEDV increased significantly in the SDB group over 12 weeks, while it remained 
TABLE 1 Baseline patient characteristics

\begin{tabular}{|c|c|c|c|}
\hline & SDB & No SDB & p-value \\
\hline Subjects $\mathrm{n}$ & 29 & 25 & \\
\hline Age years & $55 \pm 10$ & $53 \pm 10$ & 0.563 \\
\hline $\mathrm{BMI} \mathrm{kg} \cdot \mathrm{m}^{-2}$ & $30 \pm 4$ & $27 \pm 3$ & 0.002 \\
\hline Male & $26(90)$ & $19(76)$ & 0.179 \\
\hline Hypertension & $16(55)$ & $15(60)$ & 0.721 \\
\hline Diabetes mellitus & $5(17)$ & $3(12)$ & 0.589 \\
\hline Hypercholesterolaemia & $9(31)$ & $10(40)$ & 0.492 \\
\hline Smoking & $19(66)$ & $22(88)$ & 0.054 \\
\hline STEMI & $24(83)$ & $20(80)$ & 0.856 \\
\hline Target of primary coronary occlusion & & & 0.363 \\
\hline LAD & $14(48)$ & $9(36)$ & \\
\hline Non-LAD & $15(52)$ & $16(64)$ & \\
\hline Grade 0 TIMI flow before $\mathrm{PCI}$ & $25(86)$ & $19(76)$ & 0.340 \\
\hline Grade 3 TIMI flow after PCI & 27 (93) & $24(96)$ & 0.643 \\
\hline Killip classification & & & 0.715 \\
\hline Class I & $22(76)$ & $20(80)$ & \\
\hline Class II & $7(24)$ & $5(20)$ & \\
\hline Heart rate beats $\cdot \min ^{-1}$ & $76 \pm 17$ & $71 \pm 17$ & 0.300 \\
\hline Systolic blood pressure mmHg & $130 \pm 20$ & $129 \pm 23$ & 0.875 \\
\hline Diastolic blood pressure $\mathrm{mmHg}$ & $78 \pm 12$ & $80 \pm 13$ & 0.580 \\
\hline LVEF after $\mathrm{PCl} \%$ & $45 \pm 9$ & $49 \pm 8$ & 0.057 \\
\hline LVESV after $\mathrm{PCI} \mathrm{mL}$ & $97 \pm 31$ & $77 \pm 22$ & 0.010 \\
\hline Peak CK U.L $\mathrm{L}^{-1}$ & $2023 \pm 1243$ & $1475 \pm 1271$ & 0.123 \\
\hline \multicolumn{4}{|l|}{ Medication after $\mathrm{PCl}$} \\
\hline Aspirin & $29(100)$ & $25(100)$ & 1.0 \\
\hline ADP receptor inhibitors & $29(100)$ & $25(100)$ & 1.0 \\
\hline$\beta$-blockers & 27 (93) & $25(100)$ & 0.181 \\
\hline ACE inhibitor/ARB & $29(100)$ & $25(100)$ & 1.0 \\
\hline Statins & 27 (93) & $25(100)$ & 0.181 \\
\hline \multicolumn{4}{|l|}{ Medication at 12 weeks } \\
\hline Aspirin & $26(90)$ & $24(96)$ & 0.384 \\
\hline ADP receptor inhibitors & $22(76)$ & $22(88)$ & 0.261 \\
\hline$\beta$-blockers & $25(86)$ & $22(88)$ & 0.848 \\
\hline ACE inhibitor/ARB & 27 (93) & $21(84)$ & 0.297 \\
\hline Statins & 27 (93) & $24(96)$ & 0.651 \\
\hline
\end{tabular}

Data are presented as mean \pm SD or $n(\%)$, unless otherwise stated. SDB: sleep disordered breathing; BMI: body mass index; STEMI: ST-segment elevation myocardial infarction; LAD: left anterior descending; TIMI: thrombolysis in myocardial infarction; $\mathrm{PCl}$ : percutaneous coronary intervention; LVEF: left ventricular ejection fraction; LVESV: left ventricular end-systolic volume; CK: creatinine kinase; ADP: adenosine diphosphate; ACE: angiotensin-converting enzyme; ARB: angiotensin receptor blocker.

\begin{tabular}{|c|c|c|c|}
\hline & SDB & No SDB & p-value \\
\hline Subjects n & 29 & 25 & \\
\hline AHI events $\cdot h^{-1}$ & $30(21-40)$ & $5(3-7)$ & $<0.001$ \\
\hline Central $\mathrm{AHI}$ events $\cdot \mathrm{h}^{-1}$ & $8(5-22)$ & $1(0-3)$ & 0.001 \\
\hline Oxygen desaturation index events $\cdot \mathrm{h}^{-1}$ & $21(12-31)$ & $4(2-6)$ & $<0.001$ \\
\hline Minimum oxygen saturation $\%$ & $83 \pm 7$ & $87 \pm 4$ & 0.010 \\
\hline Sleep efficiency \% & $72 \pm 13$ & $70 \pm 16$ & 0.605 \\
\hline N3 \% & $14 \pm 8$ & $17 \pm 8$ & 0.137 \\
\hline REM sleep \% & $14 \pm 6$ & $18 \pm 8$ & 0.047 \\
\hline
\end{tabular}

Data are presented as median (interquartile range) or mean $\pm \mathrm{SD}$, unless otherwise stated. SDB: sleep disordered breathing; AHI: apnoea-hypopnoea index; N3: slow wave sleep; REM: rapid eye movement. 
TABLE 3 Changes in right ventricular morphological and functional parameters

\begin{tabular}{|c|c|c|c|}
\hline & SDB & No SDB & p-value \\
\hline Subjects $\mathrm{n}$ & 29 & 25 & \\
\hline \multicolumn{4}{|l|}{ RVEDV mL } \\
\hline Baseline & $129 \pm 28$ & $132 \pm 28$ & 0.716 \\
\hline 12 weeks & $143 \pm 29$ & $132 \pm 31$ & 0.200 \\
\hline$\Delta$ & $14 \pm 23$ & $0 \pm 17$ & 0.020 \\
\hline p-value ${ }^{\#}$ & 0.003 & 0.954 & \\
\hline \multicolumn{4}{|c|}{ RVEDV index $\mathrm{mL} \cdot \mathrm{m}^{-2}$} \\
\hline Baseline & $62 \pm 13$ & $68 \pm 13$ & 0.099 \\
\hline 12 weeks & $69 \pm 14$ & $68 \pm 13$ & 0.777 \\
\hline$\Delta$ & $7 \pm 11$ & $0 \pm 9$ & 0.016 \\
\hline p-value $\#$ & 0.004 & 0.861 & \\
\hline \multicolumn{4}{|l|}{ RVESV mL } \\
\hline Baseline & $56 \pm 21$ & $53 \pm 16$ & 0.648 \\
\hline 12 weeks & $58 \pm 21$ & $51 \pm 15$ & 0.162 \\
\hline$\Delta$ & $3 \pm 14$ & $-2 \pm 11$ & 0.179 \\
\hline p-value $\#$ & 0.276 & 0.411 & \\
\hline \multicolumn{4}{|c|}{ RVESV index $\mathrm{mL} \cdot \mathrm{m}^{-2}$} \\
\hline Baseline & $27 \pm 10$ & $28 \pm 8$ & 0.747 \\
\hline 12 weeks & $28 \pm 10$ & $27 \pm 7$ & 0.493 \\
\hline$\Delta$ & $1 \pm 7$ & $-1 \pm 6$ & 0.163 \\
\hline$p$-value $\#$ & 0.280 & 0.367 & \\
\hline \multicolumn{4}{|l|}{ RVEF $\%$} \\
\hline Baseline & $58 \pm 10$ & $60 \pm 8$ & 0.466 \\
\hline 12 weeks & $59 \pm 9$ & $61 \pm 8$ & 0.520 \\
\hline$\Delta$ & $2 \pm 5$ & $1 \pm 5$ & 0.766 \\
\hline p-value $\#$ & 0.081 & 0.248 & \\
\hline \multicolumn{4}{|l|}{ TAPSE cm } \\
\hline Baseline & $2.1 \pm 0.5$ & $2.2 \pm 0.4$ & 0.234 \\
\hline 12 weeks & $2.2 \pm 0.3$ & $2.2 \pm 0.4$ & 0.878 \\
\hline$\Delta$ & $0.2 \pm 0.4$ & $0.0 \pm 0.4$ & 0.150 \\
\hline p-value & 0.040 & 0.992 & \\
\hline
\end{tabular}

Data are presented as mean \pm SD, unless otherwise stated. SDB: sleep disordered breathing; RVEDV: right ventricular end-diastolic volume; RVESV: right ventricular end-systolic volume; RVEF: right ventricular ejection fraction; TAPSE: tricuspid annular plane systolic excursion. \#: baseline versus 12 weeks.

unchanged in the no SDB group (table 3 and fig. 2). The increase in RVEDV from baseline to 12 weeks was significantly greater in the SDB compared with the no SDB group (table 3). This effect persisted after accounting for LVESV or body surface area (table 3). There was no significant change in RVESV in either group (table 3). RVEF and TAPSE were similar in the SDB and no SDB groups at baseline and after 12 weeks, and changes from baseline to 12 weeks did not differ significantly between groups (table 3 ).

FIGURE 2 Change in right ventricular volumes. Data are presented as mean \pm SE. SDB: sleep disordered breathing; RVEDV: right ventricular end-diastolic volume; RVESV: right ventricular end-systolic volume.

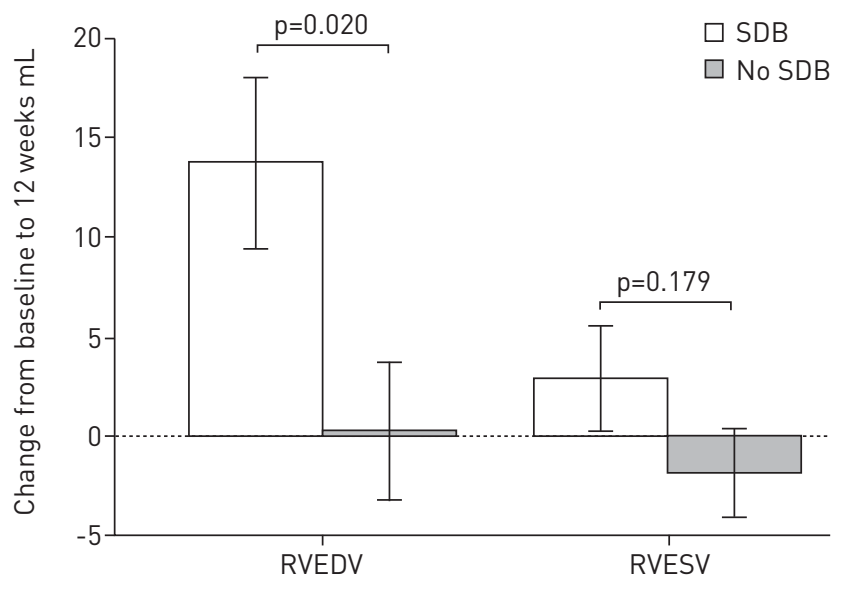


The change in RVEDV and RVEDV index from baseline to 12 weeks was similar in patients with OSA and CSA (table 4).

The analysis was repeated in a subset of patients with RV involvement of myocardial infarction $(\mathrm{n}=24$, $44 \%$ of the study population), of whom $54 \%$ had SDB $(n=13)$ and $46 \%$ had no SDB $(n=11)$. As with the primary analysis, RVEDV and RVESV were similar in the SDB group and the no SDB group $(124 \pm 37 \mathrm{~mL}$ versus $137 \pm 32 \mathrm{~mL}, \mathrm{p}=0.379$, and $52 \pm 23 \mathrm{~mL}$ versus $55 \pm 19 \mathrm{~mL}, \mathrm{p}=0.668$, respectively). In patients with $\mathrm{SDB}$, RVEDV increased significantly from baseline to 12 weeks (an increase of $14 \pm 20 \mathrm{~mL}, \mathrm{p}=0.029$ ); there was no significant change in the no SDB group $(-3 \pm 20 \mathrm{~mL}, \mathrm{p}=0.640)$. In addition, there was no significant difference in the infarct size of the right ventricle between the no SDB group and the SDB group $(6.7 \pm 1.7$ $\mathrm{mL}$ versus $5.8 \pm 2.2 \mathrm{~mL}, \mathrm{p}=0.301$ ).

\section{Right atrial parameters}

Right atrial diastolic area (RA-DA) increased in both patient groups during the 12 weeks following AMI (fig. 3). The change in RA-DA from baseline to 12 weeks was similar in patients with OSA and CSA (table 4). The increase in RA-DA was significantly larger in the SDB group compared with the no SDB group (table 5 and fig. 3). This difference was attenuated after accounting for LVESV $(p=0.166)$. There was no significant difference between groups in changes in right atrial systolic area (RA-SA) or right atrial fractional area.

\section{Linear regression models}

In the unadjusted linear regression analysis, AHI was significantly associated with RVEDV and RA-SA, but not RVESV and RA-DA (table 6). In the fully adjusted multivariable linear regression analysis, accounting for age, sex, smoking, BMI, LVESV and LV mass, AHI was still significantly associated with RVEDV ( $p=0.041$ ). AHI was not significantly associated with RVESV, RA-DA and RA-SA in the fully adjusted model.

TABLE 4 Changes in right ventricular and atrial morphological parameters in obstructive sleep apnoea (OSA) and central sleep apnoea (CSA)

\begin{tabular}{lccc} 
& OSA & CSA & p-value \\
\hline Subjects $\mathbf{n}$ & 15 & 14 & \\
RVEDV $\mathbf{~ L L}$ & $17 \pm 22$ & $11 \pm 3.6$ & 0.492 \\
RVEDV index $\mathbf{~ m L} \cdot \mathbf{m}^{-2}$ & $8 \pm 11$ & $5 \pm 12$ & 0.561 \\
RVESV $\mathbf{~ m L}$ & $4 \pm 14$ & $1 \pm 15$ & 0.583 \\
RVESV index $\mathbf{~ m L} \cdot \mathbf{m}^{-2}$ & $2 \pm 3.6$ & $1 \pm 8$ & 0.634 \\
RA-DA cm $^{2}$ & $3.1 \pm 3.0$ & $2.7 \pm 4.6$ & 0.807 \\
RA-SA cm & $1.7 \pm 2.2$ & $2.3 \pm 3.7$ & 0.572
\end{tabular}

Data are presented as mean \pm SD, unless otherwise stated. RVEDV: right ventricular end-diastolic volume; RVESV: right ventricular end-systolic volume; RA-DA: right atrial diastolic area; RA-SA: right atrial systolic area.

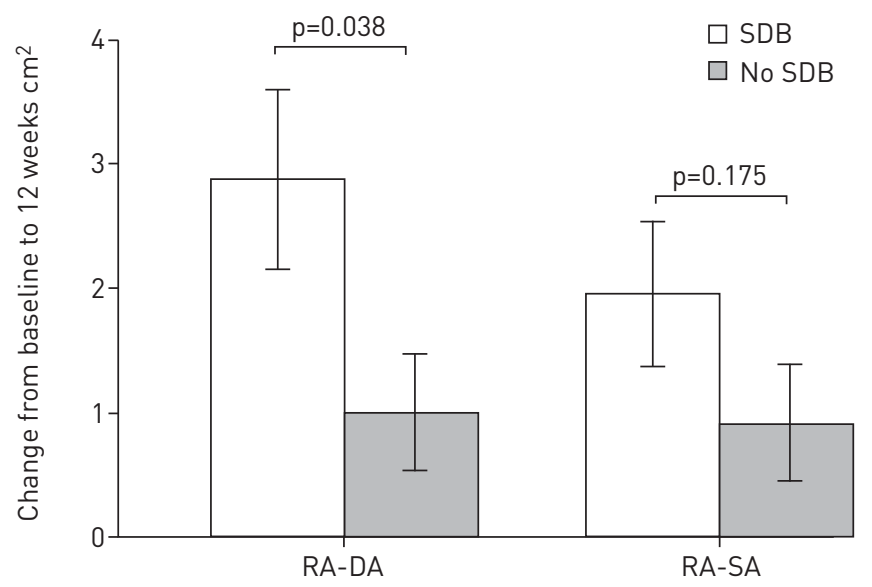

FIGURE 3 Change in right atrial area. Data are presented as mean \pm SE. SDB: sleep disordered breathing; RA-DA: right atrial diastolic area; RA-SA: right atrial systolic area. 


\begin{tabular}{|c|c|c|c|}
\hline & SDB & No SDB & p-value \\
\hline Subjects n & 27 & 25 & \\
\hline \multicolumn{4}{|l|}{ RA-DA $\mathrm{cm}^{2}$} \\
\hline Baseline & $25.3 \pm 4.1$ & $25.5 \pm 3.6$ & 0.877 \\
\hline 12 weeks & $28.1 \pm 4.4$ & $26.5 \pm 4.1$ & 0.194 \\
\hline$\Delta$ & $2.9 \pm 3.7$ & $1.0 \pm 2.4$ & 0.038 \\
\hline p-value $e^{\#}$ & 0.001 & 0.048 & \\
\hline \multicolumn{4}{|l|}{ RA-SA $\mathrm{cm}^{2}$} \\
\hline Baseline & $18.3 \pm 3.6$ & $17.6 \pm 2.7$ & 0.437 \\
\hline 12 weeks & $20.0 \pm 4.1$ & $18.5 \pm 3.0$ & 0.160 \\
\hline$\Delta$ & $2.0 \pm 3.0$ & $0.9 \pm 2.4$ & 0.175 \\
\hline p-value $\#$ & 0.002 & 0.063 & \\
\hline \multicolumn{4}{|l|}{ RA-FAC \% } \\
\hline Baseline & $27.4 \pm 10.7$ & $30.7 \pm 7.9$ & 0.214 \\
\hline 12 weeks & $29.1 \pm 8.4$ & $30.0 \pm 6.0$ & 0.657 \\
\hline$\Delta$ & $0.8 \pm 11.8$ & $-0.8 \pm 8.1$ & 0.577 \\
\hline$p$-value & 0.721 & 0.641 & \\
\hline
\end{tabular}

Data are presented as mean $\pm \mathrm{SD}$, unless otherwise stated. SDB: sleep disordered breathing; RA-DA: right atrial diastolic area; RA-SA: right atrial systolic area; RA-FAC: right atrial fractional area change. " baseline versus 12 weeks.

\section{Discussion}

The current study provides novel insights into right heart remodelling in patients with SDB early after AMI. The results show that patients with AMI and SDB had a greater increase in RVEDV within the 12 weeks following AMI compared with those without SDB. In addition, RA-DA increased to a significantly greater extent in AMI patients with SDB versus patients without SDB over the 12-week follow-up period. Measures of RV function were similar and did not change over 12-week follow-up in AMI patients with or without SDB. OSA and CSA are associated with similar changes of RV and atrial morphological parameters in the early phase after AMI.

The finding that RVEDV increases in AMI patients with SDB is supported by two recent interventional studies, both of which also used CMR to assess RV remodelling in patients with SDB and non-cardiac disease $[14,15]$. These studies showed that treatment of SDB with continuous positive airway pressure decreased RV volume, suggesting that SDB has a negative influence on the right ventricle even in the absence of other cardiovascular conditions. The magnitude of the decrease in RVEDV index after treatment of SDB in these interventional studies $\left(10 \mathrm{~mL} \cdot \mathrm{m}^{-2}\right.$ in [14] and $8 \mathrm{~mL} \cdot \mathrm{m}^{-2}$ in [15]) was comparable to the increase in RVEDV index seen in patients with SDB after AMI in the present study $\left(7 \mathrm{~mL} \cdot \mathrm{m}^{-2}\right)$. To our knowledge, this is the first study showing changes in the right ventricle after AMI in patients with SDB.

The question arises as to whether changes in RV volume are directly caused by SDB or by another factor, such as LV function. However, the increase of RVEDV from baseline to 12 weeks remained significantly

TABLE 6 The relationship between apnoea-hypopnoea index (AHI) and the change in right ventricular volumes and right atrial areas: linear regression models

\begin{tabular}{|c|c|c|c|c|c|}
\hline \multicolumn{2}{|c|}{ Variables } & \multicolumn{2}{|c|}{ Unadjusted } & \multicolumn{2}{|c|}{ Adjusted $^{\#}$} \\
\hline RVEDV & $\mathrm{AHI}$ & $0.324(0.035-0.612)$ & 0.028 & $0.315(0.013-0.617)$ & 0.041 \\
\hline RA-DA & $\mathrm{AHI}$ & $0.041(-0.005-0.087)$ & 0.082 & $-0.006(-0.054-0.041)$ & 0.790 \\
\hline RA-SA & $\mathrm{AHI}$ & $0.038(0.000-0.077)$ & 0.049 & $0.017(-0.023-0.054)$ & 0.398 \\
\hline
\end{tabular}

RVEDV: right ventricular end-diastolic volume; RVESV: right ventricular end-systolic volume; RA-DA: right atrial diastolic area; RA-SA: right atrial systolic area. ${ }^{\#}$ : adjusted for age, sex, body mass index, smoking, left ventricular end-systolic volume and left ventricular mass. 
greater in AMI patients with SDB versus patients without SDB after accounting for LVESV, a marker of LV function. This is reinforced by the fact that, in accordance with previous studies [20, 21], ZorNOFF et al. [1] also concluded that RV dysfunction is largely independent of LV function. In addition, the significant association of AHI and RVEDV was robust after accounting for age, sex, smoking, BMI, LVESV and LV mass. Furthermore, because previous studies have suggested that RV dysfunction might be more susceptible to the influence of the location and magnitude of myocardial infarction than to the extent of LV dysfunction [1, 22], we conducted an analysis based on myocardial infarct location in both patient groups. This showed that RV involvement was seen in both the SDB and the no SDB groups across a range of infarct locations. Secondly, we conducted a subanalysis of patients with proven RV involvement on CMR, which showed similar results to the main analysis of all patients. There was also no significant difference in terms of the target of primary coronary occlusion between both groups. In summary, this suggests that enlargement of the right ventricle after AMI in SDB patients is independent of LV function, RV involvement in AMI and differing sites of primary coronary artery occlusion. The results of this study cannot definitely prove that SDB is the only cause of an increase in RV volume. However, taking into account data from previous interventional studies $[14,15]$, our data provide good evidence to support the conclusion that SDB contributes to the observed effects.

In a similar manner to the changes in RVEDV, there was a significantly greater increase in RA-DA in AMI patients with SDB compared to those without SDB. This finding expands on the results of previous studies and has not been assessed previously.

RVEF, as a measure of RV contractility, was similar in those with and without SDB and remained unchanged in both groups over the follow-up period. This is in line with previous research showing no significant changes in RVEF after 12 weeks [14], or 6 and 12 months [15], of continuous positive airway pressure therapy in patients with SDB and without known heart disease.

LA-SA was significantly increased in AMI patients with SDB, while left atrial fractional area change was impaired in this group, suggesting that contractility of the left atrium is lower in patients with SDV versus patients without SDB. Such data complement previous studies showing an increased left atrial area in patients with SDB and without known cardiac disease [23, 24].

There are a number of pathogenic mechanisms that might explain the results observed in this study. Patients with SDB are exposed to chronic intermittent hypoxia, leading to pulmonary hypertension due to hypoxic vasoconstriction [10-12, 25] and reduced pulmonary levels of nitric oxide [13], in turn resulting in increased afterload of the right ventricle. Mild levels of pulmonary hypertension have been reported to occur in up to $20 \%$ of patients with OSA without pre-existing pulmonary or cardiovascular conditions [26, 27]. Increased preload of the right ventricle is caused by elevated negative intrathoracic pressure occurring during exaggerated inspirational efforts due to obstructive apnoeas; this leads to increased venous return, volume overload and subsequent distension of the right ventricle [28]. While the mechanism of pulmonary hypertension due to intermittent hypoxia in OSA may be extrapolated to some extent to CSA, the mechanism of increased RV preload due to negative intrathoracic pressure swings is a phenomenon of obstructive apnoea. Nevertheless, our data do not support the idea that OSA causes greater RV structural changes than CSA. Furthermore, myocardial infarction may itself cause RV dysfunction. Impaired RV function after AMI is often associated with LV dysfunction, which is common after AMI [29].

LV dysfunction may increase left atrial pressure leading to left atrial enlargement [23]. RV function is highly dependent on afterload, for which left atrial pressure is a widely accepted indicator [1,30]. This fact is supported by the current results, which show RV enlargement in the presence of increased left atrial area.

The results of this study support the clinically important evidence that SDB may have specific adverse effects in patients in the early stages following AMI, not only on the left heart but also on the right heart. Increased focus on such deleterious changes could help to improve strategies for the prevention of impaired long-term cardiovascular function after AMI.

This study does have some limitations. Both CSA and OSA can be temporarily worsened during the acute phase of AMI [31] and improvement of CSA and OSA in the early stages following AMI is associated with an improvement in cardiac function [6]. The observational study design cannot account for the change of SDB burden overtime in the early stages following AMI and cannot prove causal relationships (between CSA and OSA and RV remodelling, for example). Larger interventional trials are therefore required to test whether the observed effects can be partially reversed with CSA and OSA treatment in AMI patients.

In conclusion, the findings of this study suggest that SDB diagnosed shortly after AMI predicts an enlargement of the right heart within the following 12 weeks, marked by an increase in RV volume, independent of LV function or size of RV infarction in AMI. 


\section{Acknowledgements}

The authors would like to thank Astrid Brandl-Novak (Klinik und Poliklinik für Innere Medizin II, Universitätsklinikum Regensburg, Regensburg, Germany), Astrid Braune (Klinik und Poliklinik für Innere Medizin II), Ruth Luigart (Klinik und Poliklinik für Innere Medizin II) and Katja Ziczinski (Institut für Röntgendianostik, Universitätsklinikum Regensburg) for excellent assistance. English language editing assistance was provided by Nicola Ryan, an independent medical writer, funded by ResMed Germany.

\section{References}

1 Zornoff LA, Skali H, Pfeffer MA, et al. Right ventricular dysfunction and risk of heart failure and mortality after myocardial infarction. J Am Coll Cardiol 2002; 39: 1450-1455.

2 Sakata K, Yoshino H, Kurihara $\mathrm{H}$, et al. Prognostic significance of persistent right ventricular dysfunction as assessed by radionuclide angiocardiography in patients with inferior wall acute myocardial infarction. Am J Cardiol 2000; 85: 939-944.

3 Nakashima H, Katayama T, Takagi C, et al. Obstructive sleep apnoea inhibits the recovery of left ventricular function in patients with acute myocardial infarction. Eur Heart J 2006; 27: 2317-2322.

4 Lee $\mathrm{CH}$, Khoo SM, Tai BC, et al. Obstructive sleep apnea in patients admitted for acute myocardial infarction. Prevalence, predictors, and effect on microvascular perfusion. Chest 2009; 135: 1488-1495.

5 Skinner MA, Choudhury MS, Homan SD, et al. Accuracy of monitoring for sleep-related breathing disorders in the coronary care unit. Chest 2005; 127: 66-71.

6 Buchner S, Greimel T, Hetzenecker A, et al. Natural course of sleep-disordered breathing after acute myocardia infarction. Eur Respir J 2012; 40: 1173-1179.

7 Hetzenecker A, Buchner S, Greimel T, et al. Cardiac workload in patients with sleep-disordered breathing early after acute myocardial infarction. Chest 2013; 143: 1294-1301.

8 Hamilton GS, Solin P, Walker A. Coronary blood flow becomes uncoupled from myocardial work during obstructive sleep apnea in the presence of endothelial dysfunction. Sleep 2008; 31: 809-816.

9 Buchner S, Satzl A, Debl K, et al. Impact of sleep-disordered breathing on myocardial salvage and infarct size in patients with acute myocardial infarction. Eur Heart J 2014; 35: 192-199.

10 Arias MA, Garcia-Rio F, Alonso-Fernandez A, et al. Pulmonary hypertension in obstructive sleep apnoea: effects of continuous positive airway pressure: a randomized, controlled cross-over study. Eur Heart J 2006; 27: $1106-1113$.

11 Bady E, Achkar A, Pascal S, et al. Pulmonary arterial hypertension in patients with sleep apnoea syndrome. Thorax 2000; 55: 934-939.

12 Bonsignore MR, Marrone O, Insalaco G, et al. The cardiovascular effects of obstructive sleep apnoeas: analysis of pathogenic mechanisms. Eur Respir I 1994; 7: 786-805.

13 Lattimore JD, Wilcox I, Adams MR, et al. Treatment of obstructive sleep apnoea leads to enhanced pulmonary vascular nitric oxide release. Int J Cardiol 2008; 126: 229-233.

14 Magalang UJ, Richards K, McCarthy B, et al. Continuous positive airway pressure therapy reduces right ventricular volume in patients with obstructive sleep apnea: a cardiovascular magnetic resonance study. J Clin Sleep Med 2009; 5: 110-114.

15 Colish J, Walker JR, Elmayergi N, et al. Obstructive sleep apnea: effects of continuous positive airway pressure on cardiac remodeling as assessed by cardiac biomarkers, echocardiography, and cardiac MRI. Chest 2012; 141: $674-681$.

16 Sleep-related breathing disorders in adults. Recommendations for syndrome definition and measurement techniques in clinical research. The Report of an American Academy of Sleep Medicine Task Force. Sleep 1999; 22: 667-689.

17 Iber C. American Academy of Sleep Medicine. The AASM manual for the scoring of sleep and associated events: Rules, terminology and technical specifications. Westchester, American Academy of Sleep Medicine, 2007

18 Nijveldt R, Germans T, McCann GP, et al. Semi-quantitative assessment of right ventricular function in comparison to a 3d volumetric approach: a cardiovascular magnetic resonance study. Eur Radiol 2008; 18: 2399-2405.

19 Maldonado G, Greenland S. Simulation study of confounder-selection strategies. Am J Epidemiol 1993; 138 : 923-936.

20 Pfisterer M, Emmenegger H, Soler M, et al. Prognostic significance of right ventricular ejection fraction for persistent complex ventricular arrhythmias and/or sudden cardiac death after first myocardial infarction: relation to infarct location, size and left ventricular function. Eur Heart J 1986; 7: 289-298.

21 Gadsboll N, Hoilund-Carlsen PF, Madsen EB, et al. Right and left ventricular ejection fractions: relation to one-year prognosis in acute myocardial infarction. Eur Heart J 1987; 8: 1201-1209.

22 Pfisterer M, Emmenegger H, Muller-Brand J, et al. Prevalence and extent of right ventricular dysfunction after myocardial infarction-relation to location and extent of infarction and left ventricular function. Int J Cardiol 1990; 28: $325-332$

23 Oliveira W, Campos O, Cintra F, et al. Impact of continuous positive airway pressure treatment on left atrial volume and function in patients with obstructive sleep apnoea assessed by real-time three-dimensional echocardiography. Heart 2009; 95: 1872-1878.

24 Kaviraj B, Bai SC, Su L, et al. Effect of obstructive sleep apnea syndrome on serum C-reactive protein level, left atrial size and premature atrial contraction. Nan Fang Yi Ke Da Xue Xue Bao 2011; 31: 197-200.

25 Sajkov D, Wang T, Saunders NA, et al. Daytime pulmonary hemodynamics in patients with obstructive sleep apnea without lung disease. Am J Respir Crit Care Med 1999; 159: 1518-1526.

26 Alchanatis M, Tourkohoriti G, Kakouros S, et al. Daytime pulmonary hypertension in patients with obstructive sleep apnea: the effect of continuous positive airway pressure on pulmonary hemodynamics. Respiration 2001; 68 566-572.

27 Atwood CW Jr, McCrory D, Garcia JG, et al. Pulmonary artery hypertension and sleep-disordered breathing: ACCP evidence-based clinical practice guidelines. Chest 2004; 126: 72S-77S. 
28 Malone S, Liu PP, Holloway R, et al. Obstructive sleep apnoea in patients with dilated cardiomyopathy: effects of continuous positive airway pressure. Lancet 1991; 338: 1480-1484.

29 Marmor A, Geltman EM, Biello DR, et al. Functional response of the right ventricle to myocardial infarction: dependence of the site of left ventricular infarction. Circulation 1981; 64: 1005-1011.

30 Rigolin VH, Robiolio PA, Wilson JS, et al. The forgotten chamber: the importance of the right ventricle. Cathet Cardiovasc Diagn 1995; 35: 18-28.

31 Tsukamoto K, Ohara A. Temporal worsening of sleep-disordered breathing in the acute phase of myocardial infarction. Circ J 2006; 70: 1553-1556. 